M. Itô

Nagoya Math. J.

Vol. 92 (1983), 153-161

\title{
TRANSIENT MARKOV CONVOLUTION SEMI-GROUPS AND THE ASSOCIATED NEGATIVE DEFINITE FUNCTIONS
}

\author{
MASAYUKI ITÔ \\ Dedicated to Professor Makoto Ohtsuka on the \\ occasion of his 60th birthday
}

$\S 1$. Let $X$ be a locally compact and $\sigma$-compact abelian group and $\hat{X}$ be the dual group of $X^{1}$. We denote by $\xi$ a fixed Haar measure on $X$ and by $\hat{\xi}$ the Haar measure on $\hat{X}$ associated with $\xi$. It is well-known that (see, for example, [1]):

(A) For a sub-Markov convolution semi-group $\left(\alpha_{t}\right)_{t \geqq 0}$ on $X$, there exists a uniquely determined negative definite function $\psi$ on $\hat{X}$ such that

$$
\hat{\alpha}_{t}(\hat{x})=\exp (-t \psi(\hat{x})) \quad \text { for any } \hat{x} \in \hat{X}(t \geqq 0),
$$

where $\hat{\alpha}_{t}$ denotes the Fourier transform of $\alpha_{t}$.

(B) For a negative definite function $\psi$ on $\hat{X}$, there exists a uniquely determined sub-Markov convolution semi-group $\left(\alpha_{t}\right)_{t \geqq 0}$ on $X$ satisfying (1.1).

In this case, $\psi$ is called the negative definite function associated with $\left(\alpha_{t}\right)_{t \geq 0}$.

There is an interesting characterization of the transience of subMarkov convolution semi-groups.

THEOREM. Let $\left(\alpha_{t}\right)_{t \geqslant 0}$ be a sub-Markov convolution semi-group on $X$ and $\psi$ be the negative definite function associated with $\left(\alpha_{t}\right)_{t \geqq 0}$. Then $\left(\alpha_{t}\right)_{t \geqq 0}$ is transient if and only if $\operatorname{Re}(1 / \psi)$ is locally $\hat{\xi}$-summable, where $\operatorname{Re}(1 / \psi)$ denotes the real part of $1 / \psi$.

The "only if" part is easily seen (see, for example, [1]). But it is known only to show the "if" part by probabilistic methods (see [3]).

The purpose of this note is to give a simple and non-probabilistic proof of the "if" part.

Received October 7, 1982.

1) We denote by + the product of $X$ and that of $\hat{X}$. 
§2. We denote by:

$C_{K}(X)$ the usual topological vector space of all real-valued continuous functions on $X$ with compact support;

$M(X)$ the topological vector space of all real Radon measures on $X$ with the vague (weak*) topology;

$M_{K}(X)$ the subspace of $M(X)$ constituted by real Radon measures on $X$ with compact support;

$C_{K}^{+}(X), M^{+}(X)$ and $M_{K}^{+}(X)$ their subsets of all non-negative elements.

A family $\left(\alpha_{t}\right)_{t \geqq 0}$ in $M^{+}(X)$ is called a convolution semi-group on $X$ if $\alpha_{0}=$ the unit measure $\varepsilon$ at the origin $0, \alpha_{t} * \alpha_{s}=\alpha_{t+s}$ for all $t \geqq 0, s \geqq 0$ and the mapping $R^{+} \ni t \rightarrow \alpha_{t} \in M^{+}(X)$ is continuous, where $R^{+}$denotes the totality of non-negative numbers.

It is said to be transient if $\int_{0}^{\infty} \alpha_{t} d t \in M^{+}(X)$, which results from $\int_{0}^{\infty} d t \int f d \alpha_{t}<\infty$ for all $f \in C_{K}^{+}(X)$. Put

$$
N=\int_{0}^{\infty} \alpha_{t} d t
$$

We call it the Hunt convolution kernel on $X$ defined by $\left(\alpha_{t}\right)_{t \geqq 0}$.

A sub-Markov (resp. Markov) convolution semi-group $\left(\alpha_{t}\right)_{t \geqq 0}$ on $X$ is, by definition, a convolution semi-group on $X$ which satisfies $\int d \alpha_{t} \leqq 1$ (resp. $\left.\int d \alpha_{t}=1\right)$ for all $t \geqq 0$. In this case, we see that, for any $0<p$ $\in R^{+},\left(\exp (-p t) \alpha_{t}\right)_{t \geqq 0}$ is a transient sub-Markov convolution semi-group on $X$. Put

$$
N_{p}=\int_{0}^{\infty} \exp (-p t) \alpha_{t} d t \quad(p>0) ;
$$

$\left(N_{p}\right)_{p>0}$ is called the resolvent defined by $\left(\alpha_{t}\right)_{t \geqq 0}$, and it satisfies the resolvent equation:

$$
N_{p}-N_{q}=(q-p) N_{p} * N_{q} \quad \text { for all } p>0 \text { and } q>0 .
$$

LEmma 1. Let $\left(\alpha_{t}\right)_{t \geqq 0}$ be a sub-Markov convolution semi-group on $X$ and let $\left(N_{p}\right)_{p>0}$ be the resolvent defined by $\left(\alpha_{t}\right)_{t \geqq 0}$. Then, for any $p \geqq q>$ $0, N_{p} \ll N_{q}$, that is, for any $f, g \in C_{K}^{+}(X)$ and any $a \in R^{+}, N_{p} * f \leqq N_{q} * g+a$ on $\operatorname{supp}(f)$ implies that the same inequality holds on $X$, where supp $(f)$ denotes the support of $f$.

It is well-known that $N_{p} \ll N_{p}$ (the complete maximum principle of $N_{p}$ ) 
(see, for example, [1]). This and the resolvent equation show that $N_{p} \ll N_{q}$.

LEMma 2. Let $\left(\alpha_{t}\right)_{t \geqq 0}$ and $\left(N_{p}\right)_{p>0}$ be the same as in Lemma 1. If there exist $p>0$ and $\eta \in M^{+}(X)$ such that $N_{p} * \eta$ is defined in $M^{+}(X), \eta \geqq$ $p N_{p} * \eta$ in $X$ and $\eta \neq p N_{p} * \eta$, then $\left(\alpha_{t}\right)_{t \geq 0}$ is transient.

Proof. We write inductively $\left(p N_{p}\right)^{1}=p N_{p}$ and $\left(p N_{p}\right)^{n}=\left(p N_{p}\right)^{n-1} *\left(p N_{p}\right)$ $(n=2,3, \cdots)$. Then, for any integer $n \geqq 1$,

$$
\eta \geqq\left(\varepsilon+\sum_{k=1}^{n}\left(p N_{p}\right)^{k}\right) *\left(\eta-p N_{p} * \eta\right) .
$$

Since $\eta-p N_{p} * \eta \in M^{+}(X)$ and $\eta-p N_{p} * \eta \neq 0, \quad \sum_{n=1}^{\infty}\left(p N_{p}\right)^{n} \quad$ converges vaguely. We see easily that

$$
\int_{0}^{\infty} \alpha_{t} d t=\frac{1}{p} \sum_{n=1}^{\infty}\left(p N_{p}\right)^{n}
$$

which shows Lemma 2.

Lemma 3. Let $\left(\alpha_{t}\right)_{t \geqq 0}$ be a Markov convolution semi-group on $X$ and assume that the closed subgroup generated by $\cup_{t \geqq 0} \operatorname{supp}\left(\alpha_{t}\right)$ is equal to $X$. If $\left(\alpha_{t}\right)_{t \geqq 0}$ is not transient, then $X$ is generated by some compact neighborhood of the origin.

Proof. Let $V$ be a compact neighborhood of the origin and let $X_{V}$ denote the closed subgroup generated by $V$. We denote by $\alpha_{t, V}$ the restriction of $\alpha_{t}$ to $X_{V}$. Then we see easily that $\left(\alpha_{t, V}\right)_{t \geqq 0}$ is a sub-Markov convolution semi-group on $X_{V}$ and that $\left(\alpha_{t}\right)_{t \geqq 0}$ is transient if and only if, for any compact neighborhood $V$ of the origin, $\left(\alpha_{t, v}\right)_{t \geq 0}$ is transient. Hence there exists a compact neighborhood $V_{0}$ of the origin such that $\left(\alpha_{t, V_{0}}\right)_{t \geqq 0}$ is not transient, that is, $\left(\alpha_{t, V_{0}}\right)_{t \geq 0}$ is a Markov convolution semi-group on $X_{V_{0}}$. Consequently $\alpha_{t}=\alpha_{t, V_{0}}$ for all $t \geqq 0$. This implies that $X=X_{V_{0}}$. Thus Lemma 3 is shown.

Lemma 4 (see, for example, [1], p. 156). Let $\left(\alpha_{t}\right)_{t \geqq 0}$ be a transient subMarkov convolution semi-group on $X$. Put $N=\int_{0}^{\infty} \alpha_{t} d t$. Then $N$ satisfies the equilibrium principle, that is, for any relatively compact open set $\omega$ in $X$, there exists $\gamma \in M_{K}^{+}(X)$ such that $\operatorname{supp}(\gamma) \subset \bar{\omega}, N * \gamma=\xi$ in $\omega$ and $N * \gamma$ $\leqq \xi$ in $X$.

Here $\operatorname{supp}(\gamma)$ denotes also the support of $\gamma$. We say that $\gamma$ is an $N$-equilibrium measure of $\omega$. 
LEMma 5. Let $\left(\alpha_{t}\right)_{t \geqq 0}$ and $N$ be the same as in Lemma 4, $\omega$ a relatively compact open set in $X, \gamma$ an $N$-equilibrium measure of $\omega$. Then, for any $\sigma \in M^{+}(X)$ with $\int d \sigma \leqq 1$, any $a \in R^{+}$and any $f \in C_{K}^{+}(X)$ with $\operatorname{supp}(\check{f}) \subset \omega$,

$$
N *(a \gamma) *(\varepsilon-\sigma) * f(0) \geqq 0 .
$$

Here we denote by $\check{f}$ the function defined by $\breve{f}(x)=f(-x)$ for all $x \in X$. In fact, this follows from

$$
N *(a \gamma) *(\varepsilon-\sigma) * f(0)=a\left(\int \check{f} d \xi-\int \check{f} d N * \gamma * \sigma\right) \geqq a\left(1-\int d \sigma\right) \int \check{f} d \xi \geqq 0 .
$$

There exists a very useful result concerning the convolution equation:

Lemma 6 (see [2]). Let $\sigma \in M^{+}(X)$ with $\int d \sigma=1$ and let $\mu \in M(X)$. Assume that $\mu$ is shift-bounded, that is, for any $f \in C_{K}(X), \mu * f$ is bounded on $X$. If $\mu * \sigma=\mu$, then every point $x$ in the closed subgroup generated by $\operatorname{supp}(\sigma)$ is a period of $\mu$, that is $\mu=\mu * \varepsilon_{x}$, where $\varepsilon_{x}$ denotes the unit measure at $x$.

LEMma 7. Let $\left(\alpha_{t}\right)_{t \geqq 0}$ and $\left(N_{p}\right)_{p>0}$ be the same as in Lemma 1. If $\overline{\cup_{t \geqq 0} \operatorname{supp}\left(\alpha_{t}\right)}$ is non-compact, then $\lim _{p \rightarrow 0} p N_{p}=0^{2)}$.

Proof. Since $p \int d N_{p} \leqq 1,\left(p N_{p}\right)_{p>0}$ is vaguely bounded. Let $\lambda$ be an arbitrary vaguely accumulation point of $\left(p N_{p}\right)_{p>0}$ as $p \rightarrow 0$. Then $\int d \lambda \leqq 1$. Choose a net $\left(p_{i} N_{p_{i}}\right)_{i \in I}$ with $p_{i} \rightarrow 0$ such that $\lim _{i \in I} p_{i} N_{p_{i}}=\lambda$. Then, for any $0<p \in R^{+}$, the resolvent equation and $p \int d N_{p} \leqq 1$ give

$$
\lambda *\left(p N_{p}\right)=\lim _{i \in I}\left(p_{i} N_{p_{i}}\right) *\left(p N_{p}\right)=\lim _{i \in I}\left(p_{i}\left(N_{p_{i}}-N_{p}\right)+p_{i}{ }^{2} N_{p_{i}} * N_{p}\right)=\lambda .
$$

If $p \int d N_{p}<1$, this and $\int d \lambda \leqq 1$ give $\lambda=0$. Assume that $p \int d N_{p}=1$. Then the above lemma shows that for any $x \in \overline{\bigcup_{t \geqq 0} \operatorname{supp}\left(\alpha_{t}\right)}=\operatorname{supp}\left(p N_{p}\right)$, $\lambda=\lambda * \varepsilon_{x}$. Since $\int d \lambda \leqq 1$ and $\bigcup_{t \geqq 0} \operatorname{supp}\left(\alpha_{t}\right)$ is non-compact, we have $\lambda=0$. Thus we obtain that $\lim _{p \rightarrow 0} p N_{p}=0$.

In the case that $\bar{U}_{t \geqq 0} \operatorname{supp}\left(\alpha_{t}\right)$ is compact, the similar argument shows that $\lim _{p \rightarrow 0} p N_{p}$ exists and it is equal to 0 or a Haar measure on the compact subgroup generated by $\bigcup_{t \geqq 0} \operatorname{supp}\left(\alpha_{t}\right)$.

2) For a net $\left(\mu_{i}\right)_{i \in I} \subset M(X)$ and $\mu \in M(X)$, we write $\lim _{i \in I} \mu_{i}=\mu$ if $\left(\mu_{i}\right)_{i \in I}$ converges vaguely to $\mu$ along $I$. 
For a real Radon measure $\mu$ on $X$, we denote by $\check{\mu}$ the real Radon measure on $X$ defined by $\int f d \check{\mu}=\int \check{f} d \mu$.

LEMMA 8. Let $\left(\alpha_{t}\right)_{t \geqq 0}$ and $\left(N_{p}\right)_{p>0}$ be the same as above and let $\left(a_{p}\right)_{p>0}$ be a family of positive numbers such that $\left(a_{p} N_{p} * \check{N}_{p}\right)_{p>0}$ is vaguely bounded. Assume that the closed subgroup generated by $\bigcup_{t \geqq 0} \operatorname{supp}\left(\alpha_{t}\right)$ is equal to $X$. Take a vaguely accumulation point $\eta$ of $\left(a_{p} N_{p} * \check{N}_{p}\right)_{p>0}$ as $p \rightarrow 0$ and a net $\left(p_{i}\right)_{i \in I}$ of positive numbers with $p_{i} \rightarrow 0$ and $\lim _{i \in I} a_{p_{i}} N_{p_{i}} * \check{N}_{p_{i}}=\eta$. If, for any $q>0, \lim _{i \in I} a_{p_{i}} N_{p_{i}} * \check{N}_{q}=0$, then $\eta=0$ or $\eta$ is proportional to $\xi$.

Proof. Since $N_{p_{i}} * \check{N}_{p_{i}}$ is of positive type, for any $f \in C_{K}(X)$,

$$
\left(a_{p_{i}} N_{p_{i}} * \check{N}_{p_{i}} * f * \check{f}\right)_{i \in I}
$$

is uniformly bounded. Let $0<q \in R^{+}$. Since $q \int d N_{q} \leqq 1$, we have

$$
\lim _{i \in I} a_{p_{i}} q^{2} N_{p_{i}} * \check{N}_{p_{i}} * N_{q} * \check{N}_{q} * f * \check{f}(x)=q^{2} \eta * N_{q} * \check{N}_{q} * f * \check{f}(x)
$$

for all $f \in C_{K}(X)$ and $x \in X$, which implies that

$$
\lim _{i \in I} a_{p_{i}} q^{2} N_{p_{i}} * \check{N}_{p_{i}} * N_{q} * \check{N}_{q}=q^{2} \eta * N_{q} * \check{N}_{q}
$$

On the other hand, we have, by our assumption,

$$
\lim _{i \in I} a_{p_{i}} q^{2} N_{p_{i}} * \check{N}_{p_{i}} * N_{q} * \check{N}_{q}=\lim _{i \in I} a_{p_{i}}\left(N_{p_{i}}-N_{q}\right) *\left(\check{N}_{p_{i}}-\check{N}_{q}\right)=\eta \text {. }
$$

Thus we have

$$
\eta=q^{2} \eta * N_{q} * \check{N}_{q} .
$$

Assume that $\eta \neq 0$. Since $\eta$ is of positive type, $\eta$ is shift-bounded. Hence $q^{2} \int d N_{q} * \check{N}_{q}=1$. Evidently $\operatorname{supp}\left(N_{q}\right)=\overline{\bigcup_{t \geq 0} \operatorname{supp}\left(\alpha_{t}\right)}$ and $\operatorname{supp}\left(N_{q}\right)$ is a closed semi-group. Hence $\operatorname{supp}\left(N_{q} * \check{N}_{q}\right)=X$, and Lemma 6 gives $\eta=c \xi$ with some constant $c>0$. Thus Lemma 8 is shown.

§3. A complex valued continuous function $\psi(\hat{x})$ on $\hat{X}$ is, by definition, negative definite if $\psi(\hat{0}) \geqq 0, \psi(-\hat{x})=\overline{\psi(\hat{x})}$ and for any integer $m \geqq 1$, any $\left(\hat{x}_{j}\right)_{j=1}^{m} \subset \hat{X}$ and any $\left(\rho_{j}\right)_{j=1}^{m} \subset C$ with $\sum_{j=1}^{m} \rho_{j}=0$,

$$
\sum_{k=1}^{m} \sum_{j=1}^{m} \psi\left(\hat{x}_{j}-\hat{x}_{k}\right) \rho_{j} \bar{\rho}_{k} \leqq 0
$$

Here $\hat{0}$ denotes the origin of $\hat{X}$ and $C$ denotes the totality of complex numbers. 
Remark 9 (see, for example, [1]). Let $\psi$ be a negative definite function on $\hat{X}$. Then we have:

(1) $\operatorname{Re} \psi$ is also negative definite.

(2) $\operatorname{Re} \psi(\hat{x}) \geqq \psi(\hat{0})$ for all $\hat{x} \in \hat{X}$, that is, $\operatorname{Re} \psi(\hat{x}) \geqq 0$. So we can write $\psi(\hat{x})=|\psi(\hat{x})| \exp \left(i \theta_{\hat{x}}\right)$ with $\left|\theta_{\hat{x}}\right| \leqq \pi / 2$.

(3) Let $\alpha \in R^{+}$with $0<\alpha \leqq 1$ and put

$$
\psi^{\alpha}(\hat{x})=\left\{\begin{array}{ll}
|\psi(\hat{x})|^{\alpha} \exp \left(i \alpha \theta_{\hat{x}}\right) & \text { if } \psi(\hat{x}) \neq 0 \\
0 & \text { if } \psi(\hat{x})=0
\end{array},\right.
$$

where $\theta_{\hat{x}}=\arg \psi(\hat{x})$ with $\left|\theta_{\hat{x}}\right| \leqq \pi / 2$. Then $\psi^{\alpha}$ is negative definite.

Evidently we have the following

Remark 10. Let $\left(\alpha_{t}\right)_{t \geqq 0}$ and $\psi$ be a sub-Markov convolution semi-group on $X$ and the negative definite function associated with $\left(\alpha_{t}\right)_{t \geq 0}$. Then we have:

(1) $\psi(\hat{0})=0$ if and only if $\int d \alpha_{t}=1$ for all $t \geqq 0$.

(2) $p\left(1-p \hat{N}_{p}\right)$ converges uniformly to $\psi$ on any compact set as $p \rightarrow \infty$, where $\left(N_{p}\right)_{p>0}$ is the resolvent defined by $\left(\alpha_{t}\right)_{t \geqq 0}$.

Consequently, if $\psi(\hat{0}) \neq 0$, then $\left(\alpha_{t}\right)_{t \geq 0}$ is always transient. We remark here that $\hat{N}_{p}(\hat{x})=1 /(p+\psi(\hat{x}))$.

§4. In this paragraph, we shall show the "if" part of Theorem.

Proposition 11. Let $\left(\alpha_{t}\right)_{t \geqq 0}$ and $\psi$ be a sub-Markov convolution semigroup on $X$ and the negative definite function associated with $\left(\alpha_{t}\right)_{t \geq 0}$. If $\operatorname{Re}(1 / \psi)$ is locally $\hat{\xi}$-summable, then $\left(\alpha_{t}\right)_{t \geqq 0}$ is transient.

Proof. Evidently we may assume that $\left(\alpha_{t}\right)_{t \geqq 0}$ is a Markov convolution semi-group, that is, $\psi(\hat{0})=0$. Furthermore, we may assume also that the closed subgroup generated by $\bigcup_{t \geqq 0} \operatorname{supp}\left(\alpha_{t}\right)$ is equal to $X$ (see, [1], p. 105). For any $0<p \in R^{+}$, we put $\psi_{p}(\hat{x})=p\left(1-p \hat{N}_{p}(\hat{x})\right)$ on $\hat{X}$. Then $\psi_{p}(\hat{x})=p \psi(\hat{x}) /(p+\psi(\hat{x}))$, so that $\operatorname{Re}\left(1 / \psi_{p}\right)$ is locally $\hat{\xi}$-summable. Furthermore, we remark that $\left(\alpha_{t}\right)_{t \geqq 0}$ is transient if and only if $\sum_{n=1}^{\infty}\left(p N_{p}\right)^{n}$ converges vaguely.

Consequently, we may assume that $\psi(\hat{x})=1-\hat{\sigma}(\hat{x})$ on $\hat{X}$, where $\sigma \epsilon$ $M^{+}(X)$ with $\int d \sigma=1$ and $\operatorname{supp}(\sigma)-\operatorname{supp}(\sigma)=X^{3)}$. Then $|\psi(\hat{x})| \leqq 2$ and $\psi(\hat{x}) \neq 0$ if $\hat{x} \neq \hat{0}$.

3) For a subsets $A, B$ of $X, A-B=\{x-y ; x \in A, y \in B\}$. 
Assume that $\left(\alpha_{t}\right)_{t \geqq 0}$ is not transient. Then $X$ is non-compact, and Lemma 3 shows that $X$ is generated by a certain compact neighborhood of the origin. Hence we may assume that $X=R^{n} \times Z^{m} \times F$, where $n, m$ are integers $\geqq 0, R$ is the additive group of real numbers, $Z$ is the additive group of integers and where $F$ is a compact abelian group (see, for example, [4], p. 109). Let $\xi_{F}$ be the normalised Haar measure on $F$. By considering the canonical projection of $\alpha_{t} * \xi_{F}$ on $R^{n} \times Z^{m}$ for all $t \geqq 0$, we may assume that $X=R^{n} \times Z^{m}$. Then $\hat{X}=R^{n} \times T^{m}$, where $T^{m}$ is the $m$-dimensional torus.

Assume that $n \geqq 1$. First we shall show that $\operatorname{Re}(1 / \psi) \hat{\xi}$ is temperate. Since $|\psi(\hat{x})| \geqq a|\hat{x}|^{2}$ in a certain neighborhood of $\hat{0}$ with some constant $a>0$, there exists an integer $m \geqq 1$ such that $\left(1 /|\psi|^{2}\right)^{1 / m}$ is locally $\hat{\xi}$ summable. Here $|\hat{x}|$ denotes the distance between $\hat{x}$ and $\hat{0}$ in $R^{n} \times T^{m}$. Let $\left(\alpha_{t, m}\right)_{t \geqq 0}$ be the Markov convolution semi-group on $X$ satisfying $\widehat{\alpha_{t, m}}$ $=\exp \left(-t \psi^{1 / m}\right)$ for all $t \geqq 0$ and let $\left(N_{p, m}\right)_{p>0}$ be the resolvent defined by $\left(\alpha_{t, m}\right)_{t \geqq 0}$. Since, for any $p>0$,

$$
{\widehat{N_{p, m} * N_{p, m}}}_{p}(\hat{x})=\frac{1}{\left|p+\psi^{1 / m}(\hat{x})\right|^{2}} \quad \text { on } \hat{X}
$$

$\left(N_{p, m} * \check{N}_{p, m}\right)_{p>0}$ is vaguely bounded. This implies that $\left(\alpha_{t, m}\right)_{t \geqq 0}$ is transient. Put $N_{0, m}=\int_{0}^{\infty} \alpha_{t, m} d t$. Then $N_{0, m} * \check{N}_{0, m}$ is defined and

$$
{\widehat{N_{0, m} * \check{N}_{0, m}}}=\left(\frac{1}{|\psi|^{2}}\right)^{1 / m} \hat{\xi} \text {. }
$$

Since $(\operatorname{Re} \psi)^{1 / m}$ is bounded, $\left(\operatorname{Re} \psi / \|\left.\psi\right|^{2}\right)^{1 / m} \hat{\xi}$ is temperate. Consequently, $\left(\operatorname{Re} \psi /|\psi|^{2}\right) \hat{\xi}=\operatorname{Re}(1 / \psi) \hat{\xi}$ is temperate. Since, for any $p>0$.

$$
\frac{1}{2}\left(\hat{N}_{p}(\hat{x})+\hat{\hat{N}}_{p}(\hat{x})\right)-p \widehat{N}_{p} * \check{N}_{p}(\hat{x})=\frac{\operatorname{Re} \psi}{|p+\psi(\hat{x})|^{2}} \leqq \operatorname{Re}\left(-\frac{1}{\psi(\hat{x})}\right) \quad \text { on } \hat{X},
$$

we see that for any $f \in C_{K}^{\infty}(X),\left(\left(\frac{1}{2}\left(N_{p}+\check{N}_{p}\right)-p N_{p} * \check{N}_{p}\right) * f * \check{f}(0)\right)_{p>0}$ is bounded. Here $C_{K}^{\infty}(X)$ denotes the totality of functions $f \in C_{K}(X)$ such that for any $y \in Z^{m}$, the function $f(x, y)$ of $x$ is infinitely differentiable on $R^{n}$.

Assume that $n=0$. Then $\hat{X}$ is compact. Hence, similarly as above, we see that for any $f \in C_{K}(X),\left(\left(\frac{1}{2}\left(N_{p}+\check{N}_{p}\right)-p N_{p} * \check{N}_{p}\right) * f * \check{f}(0)\right)_{p>0}$ is bounded.

Thus, in general, there exists $f_{0} \in C_{K}^{+}(X)$ with $f_{0} \neq 0$ such that $\left(\left(\frac{1}{2}\left(N_{p}+\check{N}_{p}\right)-p N_{p} * \check{N}_{p}\right) * f_{0} * \check{f}_{0}(0)\right)_{p>0}$ is bounded. Furthermore, $\left(p N_{p} * \check{N}_{p}\right)_{p>0}$ is not vaguely bounded. Hence $\left(p N_{p} * \check{N}_{p} * f_{0} * \breve{f}_{0}(0)\right)_{p>0}$ is not bounded. Put 
$a_{p}=\left(1 / p N_{p} * \check{N}_{p} * f_{0} * \check{f}_{0}(0)\right)(p>0)$. Since $a_{p} p N_{p} * \check{N}_{p}$ is of positive type, $\left(a_{p} p N_{p} * \check{N}_{p}\right)_{p>0}$ is vaguely bounded. We choose a decreasing sequence $\left(p_{k}\right)_{k=1}^{\infty}$ such that $\lim _{k \rightarrow \infty} p_{k}=0,\left(a_{p_{k}} p_{k} N_{p_{k}} * \check{N}_{p_{k}}\right)_{k=1}^{\infty}$ converges vaguely and that $\left(a_{p_{k}}\right)_{k=1}^{\infty}$ converges decreasingly to 0 as $k \uparrow \infty$ (Remark that $X=R^{n}$ $\left.\times Z^{m}\right)$. Put $\eta=\lim _{k \rightarrow \infty} a_{p_{k}} p_{k} N_{p_{k}} * \check{N}_{p_{k}}$. Since $\int f_{0} * \check{f}_{0} d \eta=1$, Lemma 6 shows that $\eta=c \xi$ with some constant $c>0$. Since

$$
\left(\left(\frac{1}{2}\left(N_{p_{k}}+\check{N}_{p_{k}}\right)-p_{k} N_{p_{k}} * \check{N}_{p_{k}}\right) * f_{0} * \check{f}_{0}(0)\right)_{k=1}^{\infty}
$$

is bounded, we have also

$$
\lim _{k \rightarrow \infty} a_{p_{k}}\left(N_{p_{k}}+\check{N}_{p_{k}}\right)=2 c \xi \text {. }
$$

We may assume that $\left(a_{p_{k}} N_{p_{k}}\right)_{k=1}^{\infty}$ converges vaguely. Put $\lambda=\lim _{k \rightarrow \infty} a_{p_{k}} N_{p_{k}}$; then $\lim _{k \rightarrow \infty} a_{p_{k}} \check{N}_{p_{k}}=\check{\lambda}$. Hence $\lambda \neq 0$. By Lemma 1, we see easily that for any $0<p \in R^{+}, N_{p} \ll \lambda$ and $\lambda \ll \lambda$. This implies that $\lambda$ is shift-bounded and $\lambda \geqq p \lambda * N_{p}$ for all $p>0$. By Lemma 2, we have $\lambda=p \lambda * N_{p}$ for all $p>0$. This and Lemma 6 show that $\lambda$ is proportional to $\xi$, which implies $\lambda=c \xi$. Thus $\lim _{k \rightarrow \infty} a_{p_{k}} N_{p_{k}}=\lim _{k \rightarrow \infty} a_{p_{k}} \check{N}_{p_{k}}=c \xi$. We choose a relatively compact open set $\omega$ in $X$ such that $\omega \supset \operatorname{supp}\left(f_{0} * \breve{f}_{0}\right)$. Let $\gamma_{p_{k}}$ be an $\check{N}_{p^{-}}$ equilibrium measure of $\omega$ and put $\nu_{k}=\left(1 / a_{p_{k}}\right) r_{p_{k}}(k=1,2, \cdots)$. Then $\left(\nu_{k}\right)_{k=1}^{\infty}$ is vaguely bounded, and hence we may assume that it converges vaguely. Put $\nu=\lim _{k \rightarrow \infty} \nu_{k}$. Then $\int d \nu=1 / c$, that is, $\nu \neq 0$. Let $0<p$ $\in R^{+}$. Then the resolvent equation and Lemma 7 give

$$
\lim _{k \rightarrow \infty} p_{k} N_{p_{k}} * \check{N}_{p_{k}} *\left(\varepsilon-\left(p-p_{k}\right) N_{p}\right) * \nu_{k}=\lim _{k \rightarrow \infty} p_{k} N_{p} * \check{N}_{p_{k}} * \nu_{k}=0 \text {. }
$$

Lemma 5 gives

$$
\check{N}_{p_{k}} *\left(\varepsilon-\left(p-p_{k}\right) N_{p}\right) * \nu_{k} * f_{0} * \check{f}_{0}(0) \geqq 0
$$

provided with $p \geqq p_{k}$. Hence, by putting

$$
A=\sup _{q>0}\left(\frac{1}{2}\left(N_{q}+\check{N}_{q}\right)-q N_{q} * \check{N}_{q}\right) * f_{0} * \check{f}_{0}(0)
$$

we have, for $p \geqq p_{k}$,

$$
\begin{aligned}
& \left.\left(\frac{1}{2}\left(N_{p_{k}}+\check{N}_{p_{k}}\right)-p_{k} N_{p_{k}} * \check{N}_{p_{k}}\right)\right) *\left(\varepsilon-\left(p-p_{k}\right) N_{p}\right) * \nu_{k} * f_{0} * \check{f}_{0}(0) \\
& \quad \leqq 2 A \sup _{1 \leqq k<\infty} \int d \nu_{k},
\end{aligned}
$$

because $\left(\frac{1}{2}\left(N_{p_{k}}+\check{N}_{p_{k}}\right)-p_{k} N_{p_{k}} * \check{N}_{p_{k}}\right) * f_{0} * \breve{f}_{0}$ is of positive type. Letting $k \rightarrow$ $\infty$, we obtain that 


$$
N_{p} * \nu * f_{0} * \check{f}_{0}(0) \leqq 4 A \sup _{1 \leqq k<\infty} \int d \nu_{k}
$$

This implies that $\left(\int \check{\nu} * f_{0} * \breve{f}_{0} d N_{p_{k}}\right)_{k=1}^{\infty}$ is bounded, which contradicts

$$
\lim _{k \rightarrow \infty} a_{p_{k}} N_{p_{k}}=c \xi \text { and } \lim _{k \rightarrow \infty} a_{p_{k}}=0 .
$$

Thus we see that $\left(\alpha_{t}\right)_{t \geqq 0}$ is transient. This completes the proof.

\section{BIBLIOGRAPHY}

[1] C. Berg and G. Forst, Potential theory on locally compact abelian groups, SpringerVerlag, 1975.

[2] G. Choquet and J. Deny, Sur l'équation de convolution $\mu=\mu * \sigma$, C. R. Acad. Sci. Paris, 250 (1960), 4260-4262.

[ 3 ] S. C. Port and C. J. Stone, Potential theory of random walks on abelian groups, Acta Math., 122 (1969), 19-114.

[4] A. Weil, L'intégration dans les groupes topologiques et ses applications, Hermann, Paris, 1965.

\footnotetext{
Department of Mathematics

Faculty of Sciences

Nagoya University

Chikusa-ku, Nagoya 464

Japan
} 